\title{
La pregunta por la composición material como una pregunta sobre límites*
}

\author{
The question about material composition \\ as a question about boundaries
}

\author{
Por: Gonzalo Germán Núñez Erices \\ Departamento de Filosofía \\ University of Sheffield \\ Sheffield, Reino Unido \\ E-mail: g.nunezerices@sheffield.ac.uk \\ gonzalo.gne@gmail.com \\ ORCID: 0000-0002-6641-958X
}

Fecha de recepción: 30 de abril de 2018 Fecha de aprobación: 28 de agosto de 2018

Doi: 10.17533/udea.ef.n59a05

Resumen. Desde que Peter van Inwagen en su libro Material Beings (1990) planteó La Pregunta Especial por la Composición, mucho se ha escrito sobre cuáles son las condiciones para que dos o más objetos materiales compongan juntos un objeto. Este artículo propone un estudio de la composición con relación a la noción de límite. La pregunta por la composición implica trazar un límite que determine cuándo esta ocurre y cuándo no. Hay tres respuestas tradicionales: restrictivismo, universalismo y nihilismo. Mientras para el restrictivismo la composición ocurre ocasionalmente, el universalismo y nihilismo sostienen que la composición ocurre siempre o nunca respectivamente. No obstante, pese a las respuestas opuestas de estos dos últimos, hay un objetivo que los vincula: evitar la existencia de límites ontológicamente vagos para la composición material. Así, cómo tracemos un límite determinará qué tipo de entidades aceptamos como componentes del mundo.

Palabras claves: composición, límites, restrictivismo, nihilismo, universalismo, vaguedad

Abstract. Since Peter van Inwagen in his book Material Beings (1990) raised The Special Composition Question, many lines have been written about the conditions for two or more material objects to compose a further object. This paper proposes a study of the relationship between composition and the notion of boundary. The question about composition entails drawing a boundary to determine

* Este artículo forma parte de la investigación de doctorado realizada en el Departamento de Filosofía de la Universidad de Sheffield (Reino Unido) financiado por el programa Becas Chile (adjudicación año 2012) de la Comisión Nacional de Investigación Científica y Tecnológica (CONICYT) a cargo del Ministerio de Educación del Estado de Chile.

Cómo citar este artículo:

MLA: Núñez Erices, Gonzalo Germán. "La pregunta sobre la composición material como una pregunta sobre límites". Estudios de Filosofia 59 (2019): 97-120.

APA: Núñez Erices, G. G. (2019). La pregunta sobre la composición material como una pregunta sobre límites. Estudios de Filosofia, 59, 97-120.

Chicago: Gonzalo Germán Núñez. "La pregunta sobre la composición material como una pregunta sobre límites". Estudios de Filosofia n. ${ }^{\circ} 59$ (2019): 97-120. 
when it occurs and when it does not. There are three traditional answers: restrictivism, universalism, and nihilism. While according to restrictivism, composition occurs occasionally, according to universalism and nihilism, it occurs either always or never respectively. Nonetheless, despite the opposition of these two answers, both have a common target: to avoid the existence of ontologically vague boundaries for material composition. Thus, how a boundary is drawn will determine what kind of things we accept as components of the world.

Keywords: composition, boundaries, restrictivism, nihilism, universalism, vagueness

\section{Introducción}

La tarea fundamental de la ontología, de acuerdo con W. V. Quine, es contestar la pregunta ¿Qué es lo que hay? Entre todas las cosas que puede haber, una ontología del sentido común (si hay algo que pueda ser denominado de tal manera) comprende que hay un sinfín de objetos físicos los cuales están a su vez compuestos de otros objetos físicos. Así, por ejemplo, muchos átomos componen un trozo de arcilla, un ladrillo está compuesto de agregados de arcilla, y una muralla está a su vez compuesta de muchos ladrillos. Sin embargo, no aceptaríamos en este tipo de ontología cotidiana que un ladrillo y el planeta Marte componen juntos un objeto, o que la Gran Muralla China junto con el jarrón de arcilla sobre mi mesa componen juntos otro objeto. Por otro lado, sí aceptamos, junto con los astrónomos, que pese a las distancias en años luz entre muchos cuerpos celestes, estos componen galaxias. ¿Cuál es entonces el límite para determinar cuándo dos o más objetos componen algo? ¿Existe un límite que nos señale claramente cuándo muchos objetos componen un objeto? ¿Cuáles son los criterios para trazar un límite de tales características? Este artículo es acerca de límites y composición, es decir, busca establecer cuál es el rol que juega la noción de límite en las discusiones metafísicas acerca de la composición material.

En su libro Material Beings (1990), Peter van Inwagen ha planteado una pregunta que ha sido largamente discutida hasta hoy en los debates de metafísica analítica: ¿Bajo qué condiciones la composición ocurre? ¿En qué circunstancias una pluralidad de objetos son las partes genuinas que componen un todo? O bien, en términos de la cuantificación plural empleada por van Inwagen: ¿Cuándo resulta ser verdadero que $\exists y$ y que los $x s$ compongan $y$ ? Esta pregunta es llamada La Pregunta Especial por la Composición: ${ }^{1} \mathrm{Al}$ respecto, se revisarán tres tesis

1 En lo que sigue del artículo ocuparé la abreviación PSC. 
filosóficas que han respondido PSC: restrictivismo, universalismo y nihilismo. Mientras el restrictivismo es una tesis moderada en la cual la composición ocurre ocasionalmente, el universalismo y el nihilismo son tesis radicales en las que la composición ocurre siempre o nunca, respectivamente.

Este artículo explora la noción de límite comprendida por cada una de estas tesis mencionadas. Siguiendo una interpretación restrictivista de la composición, si no hay un límite claro y preciso que determine cuándo dos o más cosas efectivamente componen algo y cuándo efectivamente no lo hacen, entonces hay un límite ontológicamente vago cuya borrosidad no depende sino únicamente de la arbitrariedad de cómo es el mundo. Las tesis universalista y nihilista se resisten a la posibilidad de límites ontológicamente vagos. Por esta razón, mientras el primero mantiene que la composición no tiene límites (siempre ocurre), el segundo sostiene que la composición tiene un límite claro y estricto (nunca ocurre). Esto lleva a que mientras para un universalista el mundo está poblado de objetos compuestos por otros objetos, para un nihilista el mundo consiste únicamente de simples. ${ }^{2}$ Así, el modo como entendamos el límite para la composición determinará los elementos que constituyen nuestro inventario del mundo.

En lo que sigue, la primera parte del artículo se enfocará en explicar PSC y revisar el restrictivismo de sentido común como una respuesta espontánea o preteórica a la pregunta sobre la composición. En particular, un criterio de sentido común respecto a cuándo dos o más objetos componen otro objeto responde al modo como los límites externos de los objetos físicos (i.e., sus superficies) interactúan entre sí. La segunda parte indaga sobre las diferentes propuestas ontológicas de las ya mencionadas tres respuestas filosóficas relacionadas con la PSC. En este sentido, tanto el universalismo como el nihilismo (pese a lo contradictorio de sus posturas) sostienen sus tesis sobre los límites de la composición con el fin de evitar, entre otras cosas, que los límites que encontramos en la realidad para determinar cuándo ocurre la composición material sean vagos. La noción nihilista y universalista de límite para la composición implica diferentes respuestas a qué es lo que hay y, sin embargo, ambas vienen motivadas por desestimar la existencia de límites vagos en el mundo.

2 Un simple es un átomo mereológico, es decir, un objeto que no posee partes. 


\section{La pregunta especial por la composición}

La pregunta por la composición es, en último término, un modo de interpretar la teoría acerca de la relación entre las partes y el todo, conocida como mereología: ${ }^{3}$ ¿Es el todo un objeto distinto a la suma de las partes que lo componen? Es conocido el planteamiento de David Lewis (1991) que establece que la mereología es ontológicamente inocente. Esto es, al aceptar la existencia de varios objetos, no hay un compromiso ontológico adicional si también consideramos la existencia del objeto que se compone de ellos. La relación partes-todo resulta ser una redundancia ontológica en virtud de una relación de identidad. Esta tesis es denominada Composición como Identidad la cual sostiene que las partes son idénticas al todo; esto es, el todo no es nada sobre o por encima de las partes que lo componen. ${ }^{4}$ La inocencia de la mereología lleva a Lewis a aceptar la tesis según la cual la composición es irrestricta, i.e., cada vez que hay dos o más objetos, siempre existirá un objeto compuesto por ellos. Así, no solo existen constelaciones compuestas de muchas estrellas, sino también el objeto compuesto por Júpiter y mi copia del libro On the plurality of the worlds. Sin embargo, ninguno de ellos implica un compromiso ontológico adicional a la aceptación de la existencia de estrellas, planetas y libros.

No obstante, no todos los filósofos aceptan que la composición conlleve una relación de identidad. ¿ ¿Cómo puede haber una relación de identidad entre muchos objetos y un objeto? Las partes son muchas y el todo es uno: lo múltiple y lo uno no puede ser, por tanto, una relación de identidad, pues implicaría una violación a la Ley de Leibniz. ${ }^{6}$ En este sentido, la composición (en términos mereológicos)

3 La mereología fue propuesta por el lógico polaco Stanislaw Lesniewski (1886-1939) en un artículo llamado 'Mereology' de 1927. Más tarde, Goodman y Leonard contribuyeron en el desarrollo de la teoría con un artículo de 1940 llamado 'Calculus of Individuals'.

4 La 'inocencia' ontológica de la tesis de Composición como Identidad es una especie de conservadurismo ontológico (Hawley, 2014, p. 71). Podemos aceptar que la composición siempre ocurre sin agregar objetos compuestos a la lista de objetos de los cuales tú ya estabas comprometido con su existencia. Por el solo hecho de aceptar la existencia de la Torre Eiffel y Júpiter, tú ya has aceptado inocentemente el objeto compuesto de estos debido a tu compromiso ontológico previo. No hay, por tanto, un compromiso ontológico adicional a los que ya tienes en el momento de aceptar la existencia de ciertos objetos. De este modo, Lewis (1991) sostiene que la composición mereológica funciona como una relación de identidad en la que una colección de objetos es idéntica al objeto total compuesto por tales objetos. Esta tesis ha sido defendida por Shoemaker (1970), Wiggins (1980) y Johnston (1992).

5 Algunos de los representantes que rechazan que la composición sea una relación de identidad son Gibbard (1975), Lewis (1991) y Noonan (1993).

6 De acuerdo con la Ley de Leibniz, necesariamente, si $x$ e $y$ comparten las mismas propiedades, entonces $x$ e $y$ son idénticos, viz., $\exists \forall \mathrm{x} \forall \mathrm{y}[\forall \mathrm{P}(\mathrm{Px} \leftrightarrow \mathrm{Py}) \rightarrow \mathrm{x}=\mathrm{y}]$. La tesis de la Composición como Identidad 
no es ontológicamente inocente: si muchos objetos componen un objeto, entonces el objeto compuesto al ser uno y no múltiple es cualitativamente diferente a las partes que lo componen.

¿Cuándo ocurre la composición material? ¿Cuál puede ser el criterio para determinar cuándo esta ocurre y cuándo no? ¿Qué tipo de propiedades instanciadas por un objeto compuesto supervienen sobre la organización y disposición de las partes que lo componen? ¿Qué consecuencias ontológicas respecto a lo que hay en el mundo implica trazar un límite a la composición material? Antes de abordar este tipo de preguntas, hay que definir la composición. En este sentido, algunas definiciones y axiomas mereológicos básicos son los siguientes: ${ }^{7}$

$\boldsymbol{x}$ es una parte (propia) de $\boldsymbol{y}=_{\mathrm{df}} x$ es una parte de $y$ no al revés; o bien, cualquier cosa que se superpone con $x$ también se superpone con $y$.

$\boldsymbol{x}$ superpone $\boldsymbol{y}=_{\mathrm{df}} x$ e $y$ comparten alguna parte; o bien, dado $x, y, \mathrm{y} z, x$ superpone $y$ si y solo si $z$ es parte tanto de $x$ como de $y$.

$\boldsymbol{x}$ es una suma (o fusión) de los $y s=_{\mathrm{df}} x$ tiene todos los $y s$ como partes y no hay parte de $x$ que no superponga alguno de los $y s$.

Transitividad: dado $x, y, \mathrm{y} z$, si $x$ es parte de $z \mathrm{y} z$ es parte de $y$, entonces $x$ es parte de $y$.

Exclusividad de la composición: Nunca sucede que los mismos objetos generen dos fusiones diferentes; o dado $x, y, \mathrm{y} z$, si $x$ e $y$ componen $z$, entonces $x$ y $z$ solamente componen $z$.

Teniendo a la mano lo anterior, Van Inwagen define la composición del siguiente modo:

Los $x$ s componen $y=_{\text {df }}$ cada uno de los $x s$ es una parte de $y$, no dos de los $x s$ comparten una parte entre sí, y cada parte de y comparte una parte con al menos uno de los $x s$.

viola el espíritu de esta ley la cual expresa que la identidad cualitativa implica identidad numérica. Una propiedad básica de un todo es que es uno, mientras que lo que caracteriza a las partes es que es múltiple. Puesto que el todo y las partes son cualitativamente diferentes, entonces son numéricamente diferentes también.

7 Estas definiciones y axiomas son tomadas de Lewis (1991), Simons (1987), y Varzi (2016). 
Podemos plantear nuevamente la pregunta por la composición a partir de esta definición: ¿Bajo qué circunstancias dos o más objetos son las partes (genuinas) que componen otro objeto? Desde un punto de vista más intuitivo o pre-teórico, los límites de las cosas (las superficies) parecen jugar un rol importante en la respuesta a PSC: dos cosas componen algo más cuando sus límites o partes de sus límites están en contacto. Un conjunto de ladrillos, por ejemplo, componen una muralla cuando estos están apilados uno sobre el otro y parte de sus superficies están en contacto. Van Inwagen sugiere que el contacto es una respuesta representativa a PSC: "Para que los xs compongan algo, uno solo necesita ponerlos en contacto; si los $x s$ están en contacto, ellos componen algo; y si ellos no están en contacto, ellos no componen nada" (1990, p. 33). Podríamos decir que $x$ e $y$ están en contacto directo en el caso que no haya espacio vacío entre (partes del) límite de $x$ y (partes del) límite de $y$. Por otra parte, dado $x, y$ y $z$, los objetos $x$ e $y$ están en contacto indirecto en el caso que ninguna de las partes del límite de $x$ toque alguna parte del límite de $y$, pero $x$ e $y$ estén ambos en contacto directo con $z$. Por lo tanto, aunque la superficie de un ladrillo en la parte inferior de una muralla no está en contacto directo con la superficie de un ladrillo en la parte superior de la muralla, ambos están en contacto indirecto en virtud del contacto entre las superficies de los ladrillos que se encuentran entre ellos. Sin embargo, el contacto no es suficiente para responder PSC. Consideremos el siguiente principio:

$\exists x$ y los $y s$ componen $x$ si y solo si $\exists x$ y los $y s$ se agrupan unos con otros en virtud de una relación de contacto (ya sea directo o indirecto).

Si atendemos el sentido común, el contacto ocurre solo si dos objetos no se superponen y no hay espacio vacío entre (parte de) sus límites (el único lugar donde el contacto entre las cosas de hecho ocurre). No obstante, esto nos puede llevar situaciones controversiales en el caso de que la existencia de un límite implique una interrupción en la continuidad de la región de espacio compuesta por dos sub-regiones $R_{1}$ e $R_{2}$ ocupadas por dos objetos en contacto $x$ e $y$ respectivamente. Desde un punto de vista topológico, $x$ e $y$ no pueden estar en contacto a menos que o bien $R_{1}$ o bien $R_{2}$ sea una región espacial topológicamente abierta (i.e., una parte de su límite no figure dentro de sus componentes) mientras que la otra una región espacial topológicamente cerrada (i.e., no hay parte de su límite que no esté dentro de sus componentes). ${ }^{8}$ Como lo explica Cartwright: “[...] una región, de forma

8 La distinción topológica entre objetos abiertos y objetos cerrados proviene de Bernard Bolzano (2014) en su libro Las Paradojas del Infinito de 1851. En el parágrafo LXVI, Bolzano sugiere que cuando dos cuerpos están en contacto (i.e., cuando no hay otro cuerpo entre ellos), algunos átomos (aquello 
esférica o alguna otra, es considerada abierta solo en el caso de que ninguno de sus puntos-límite sea un miembro de ella y cerrada solo en el caso que todos sus puntos-límite sean miembros de ella" (1975, p. 154). ${ }^{9}$ Así, el contacto entre $x$ e $y$ ocurre en tanto que el límite de la región espacial que ocupa uno (el cerrado) es el complemento del límite de la región espacial que ocupa el otro (el abierto). Sin embargo, ¿cuál de las regiones de espacio es la abierta y cuál de ellas es la cerrada, $R_{1}$ o $R_{2}$ ? ¿Cuál es la que mantiene su límite y cuál no lo hace? ¿Por qué una y no la otra? Con el fin de evitar este tipo de ontología entre objetos topológicamente abiertos y objetos topológicamente cerrados, otra opción es sostener que el contacto entre $x$ e $y$ ocurre porque solo hay un límite entre $R_{l}$ e $R_{2}$. No obstante, nuevamente surge el problema de cuál de los dos debería ser poseedor de aquel único límite. Al igual que una frontera entre dos países vecinos, un límite se halla en una disputa entre dos regiones espaciales que reclaman ser su legítimo dueño.

Si no deseamos lidiar con estos problemas de arbitrariedad, entonces podemos también postular que existe un límite cuando $x$ e $y$ están en contacto el cual no pertenece a ninguno de ellos, i.e., no es parte de los elementos que componen ni a $R_{1}$ ni a $R_{2}$. Si bien está opción evita los problemas de arbitrariedad de las opciones anteriores, tiene el problema de concebir a un límite como un ente capaz de existir por sí solo. Por el contrario, un límite es tal que siempre es un 'límite de...', i.e., un límite de algo más. Los límites son entidades ontológicamente dependientes de aquellos objetos a los cuales sirven como límites. Dicho de otro modo, jamás un límite puede existir aisladamente, pues siempre es el límite de algo distinto a sí mismo. ${ }^{10}$ Por lo tanto, plantear que el

que él llama 'átomos limítrofes') pertenecen solo a uno de los dos cuerpos mientras que el otro cuerpo no posee ninguno de ellos. En otras palabras, uno de los cuerpos tiene los átomos limítrofes entre sus partes mientras que el otro no; el contacto, por ende, ocurre cuando un cuerpo es cerrado y el otro abierto (externamente delimitado por el límite del otro).

9 Para un estudio detallado de una revisión topológica de los límites revisar Varzi (1997) y Casati \& Varzi (1999; 2000).

10 La idea de un límite como un ente ontológicamente dependiente es fundamental para comprender la condición metafísica de un límite. Brentano (1988) en su libro Investigaciones Filosóficas sobre el Espacio, el Tiempo y el Continuo fue el primero en sistematizar la idea de que un límite es un ente tal que no puede existir sin ser parte de un objeto de un mayor número de dimensiones (así, un punto es límite de una línea, una línea es límite de un plano o superficie, y una superficie es límite de un cuerpo de tres dimensiones espaciales). Más tarde, Roderick Chisholm retomó la dependencia ontológica de los límites y los caracterizó como 'parásitos ontológicos' (1976, p. 51). Un 
límite entre $x$ e $y$ es una entidad ontológicamente autónoma que no pertenece a ninguna de las regiones que ocupan $R_{1}$ y $R_{2}$ respectivamente, contradice la dependencia ontológica de todo límite. Finalmente, se podría argumentar que no hay una interposición de límites entre $R_{1}$ y $R_{2}$ cuando $x$ e $y$ están contacto, sino una co-locación o coincidencia espacial: parte del límite de $R_{1}$ ocupa la misma región de espacio que parte del límite de $R_{2}$. No obstante, la co-locación espacial es una tesis metafísica que por sí misma suscita ya bastantes objeciones de las cuales es necesario hacerse cargo antes de hablar de límites ocupando una misma región espacial simultáneamente.

Las diferentes versiones del contacto como respuesta a PSC muestran diferentes tipos de dificultades. Sin embargo, el problema de esta respuesta no está en la controversia de sus versiones, sino en el hecho de que, desde una ontología de objetos ordinarios, aceptamos casos en los cuales, pese a no tener una relación de contacto, muchos objetos componen un objeto singular (e.g., constelaciones o archipiélagos). Más aún, desde un punto de vista microscópico, la mayor parte de lo que compone a los objetos físicos cotidianos como mesas, planetas y personas son grandes porciones de espacio vacío donde numerosas partículas co-existen diseminadamente. Incluso si aceptamos la tesis del contacto, esta no nos entrega una respuesta apropiada para PSC. Tal como van Inwagen argumenta en el caso cuando nos damos la mano: "A pesar de estar en contacto, nada es de tal manera que tú y yo compongamos algo. $\mathrm{O}$ bien, por lo menos, si así fuese que tú y yo componemos algo, esto no será en virtud de nuestro estar en contacto" (1990, p. 35). El contacto no solo no explica la composición, sino que al aceptarlo como la respuesta a PSC nos hace chocar nuestras cabezas contra puzles sobre límites innecesariamente.

Aparte del contacto, hay otras tres respuestas intuitivas a PSC las cuales van Inwagen llama Las Respuestas Simples de Ligazón ${ }^{11}$ (1990, pp. 56-60): fijación, cohesión, y fusión. ${ }^{12}$ En primer lugar, podemos decir que dos o más objetos componen algo cada vez que están sujetados unos a otros. A diferencia del contacto, la fijación conlleva un tipo de relación de adhesión entre los límites de los objetos. El contacto, de acuerdo con van Inwagen, es una relación altamente inestable. Para separar dos objetos que mantienen una relación de contacto tan solo necesitamos apartar sus límites hasta dejar un mínimo de espacio vacío entre ellos.

\footnotetext{
límite no existe por sí mismo, sino que su existencia requiere siempre de la existencia de algo más para lo cual el límite es límite.

11 La expresión original en inglés es 'The Simple Bonding Answers'.

12 Los conceptos que utiliza van Inwagen son fastening, cohesion, y fusion.
} 
La fijación es una relación física mucho más fuerte que podría asegurar que dos objetos que presentan tal tipo de relación componen algo. Así, dos objetos están sujetos si existe, por ejemplo, una fuerza física aplicada sobre sus superficies que los hace inseparables. Imagina la fijación magnética entre dos piezas de metal: dada la fuerza magnética entre sus superficies ellas se adhieren de modo tan fuerte que los dos objetos juntos componen algo. No obstante, tanto como el contacto, la fijación no parece ser una respuesta completa y suficiente para PSC. Van Inwagen recurre al ejemplo de darse la mano nuevamente, pero, en este caso, nuestros dedos se han entrelazado y adherido debido a una parálisis por lo que nuestras manos no están solo en contacto: ellas están fijadas entre sí. Sin embargo, esta situación no implica que tú y yo hemos compuesto algún objeto: "nuestra parálisis no ha agregado elementos al mundo; simplemente ha limitado la posibilidad de estos de reagruparse" (van Inwagen, 1990, p. 58).

Se pueden realizar objeciones similares acerca de la cohesión y fusión. Si conozco el mecanismo para desmantelar el tipo de fijación entre dos objetos, entonces esta podría no ser lo suficientemente fuerte como para traer a la existencia un objeto compuesto. Como una medida desesperada podemos decir que, con el fin de hacer que varias cosas compongan una, solo necesitamos cohesionarlas al pegar entre sí partes de sus superficies. Incluso, como plantea van Inwagen, podríamos intentar una cohesión más fuerte:

\footnotetext{
Entre objetos que han sido causados para simplemente estar cohesionados hay un límite discernible: una juntura soldada, por ejemplo, o una capa de pegamento seco. Es posible causar una unión más íntima de objetos al fundir uno con otro de tal manera que no haya un límite que pueda ser descubierto entre ellos (1990, p. 59).
}

Pareciera que para hacer que varias cosas compongan una cosa solo se necesita encontrar la manera en que los límites entre ellas sean lo menos discernibles posible. La fusión podría ser una mejor respuesta a PSC en la medida que implica que los límites entre las partes que componen algo se funden de tal modo que sea indeterminado cuándo una parte termina y cuándo la otra comienza. La composición, de este modo, ocurre cada vez que los límites entre las partes de un objeto se funden hasta desaparecer. Van Inwagen, por ejemplo, plantea el caso de dos siameses artificiales: Alicia y Beatriz. En términos teóricos, a través de una cirugía exhaustiva, se podría unir una parte de la piel de Alicia con una parte de la piel de Beatriz. Dada la precisión de la juntura y la capacidad de sanación de ambas, podría no existir un límite distinguible entre los cuerpos de Alicia y Beatriz. Sin embargo, la fusión tampoco responde adecuadamente PSC. Siguiendo el argumento de van 
Inwagen, aunque Alicia exactamente se ajusta dentro de la región espacial $\mathrm{R}_{1} \mathrm{y}$ Beatriz exactamente se ajusta dentro de la región espacial $\mathrm{R}_{2}$, no existe un objeto que exactamente se ajuste a la región espacial que sea la fusión entre $\mathrm{R}_{1}$ y $\mathrm{R}_{2}$. $\mathrm{O}$ bien, por lo menos, si existe tal objeto, este no existe en virtud de la fusión entre Alicia y Beatriz. Si Alicia y Beatriz componen un objeto cada vez que ellas existen, entonces ellas componen exactamente el mismo objeto; pero si Alicia y Beatriz componen el mismo objeto necesariamente, entonces su fusión es una explicación insuficiente de cómo la composición acontece.

Los límites son relevantes cuando pensamos en la composición. Podemos notar que todas las Repuestas Simples de Ligazón pueden ser pensadas en términos de interacciones físicas o relaciones espaciales entre los límites exteriores o superficies de las cosas. Varios objetos físicos podrían así componer un objeto cuando: (i) sus límites están en contacto hasta no dejar espacio vacío entre ellos; (ii) sus límites tienen determinadas formas o características físicas o bien existe una fuerza física entre ellos que hace que los objetos se fijen unos con otros; (iii) sus límites están tan fuertemente atados (o pegados) generando una cohesión entre ellos; o quizás, finalmente, (iv) sus límites están completamente fundidos hasta fusionar un objeto con otro. No obstante, ninguna de estas cuatro posibilidades -inspiradas en una reflexión de sentido común acerca de cómo la composición ocurre - realmente responde a PSC satisfactoriamente. Tal vez, esta pregunta pueda únicamente ser resuelta si consideramos dos respuestas extremas en las cuales la composición o bien ocurre siempre en todas las circunstancias o bien nunca, sin importar las circunstancias.

\section{Tres respuestas a la pregunta por la composición}

\section{Restrictivismo}

PSC puede tener básicamente tres respuestas distintas: siempre, nunca y ocasionalmente. Comparado con siempre y nunca, ocasionalmente es considerado como una respuesta moderada. Esta conlleva que la composición estará restringida a ciertas condiciones particulares; a esto se debe que esta respuesta ha sido comúnmente llamada Restrictivismo Mereológico o Restrictivismo Composicional (o simplemente Restricitivismo). Sostener que la composición solo ocurre en ciertas ocasiones requiere una explicación de los criterios de cuándo la composición ocurre 
y cuándo no ocurre. El sentido común es una especie de restrictivismo pre-teórico: hay casos donde la composición claramente ocurre, otros donde claramente no ocurre, y otros también donde no es tan claro si ocurre o no. ${ }^{13}$ Sin embargo, las razones para determinar los criterios de cada una de estas ocurrencias resultan ser vagas en muchos casos pues dependen de preferencias arbitrarias o simples intuiciones espaciales respecto, por ejemplo, cuán lejos o cerca las cosas requieren estar como para componer algo.

Peter van Inwagen opta por un restrictivismo filosófico más sofisticado. Su respuesta a PSC es que dos (o más) objetos componen un tercer objeto si y solo si lo que componen involucra vida; o bien, más precisamente: los $x s$ componen $y$ si y solo si $y$ es un organismo y la actividad de los $x s$ contribuye a la vida de $y$ (1990, p. 91). Básicamente, el listado ontológico de van Inwagen solo incluye simples y organismos. ${ }^{14}$ Los objetos restantes que el sentido común está cotidianamente acostumbrado a incluir en la ontología de objetos físicos no existen. Así, por ejemplo, objetos como mesas, sillas, edificios, planetas, televisores no componen el inventario de objetos físicos existentes. De acuerdo con el restrictivismo de van Inwagen, objetos sin partes y objetos compuestos que constituyan una actividad biológica son los únicos elementos de una ontología de objetos físicos. De este modo, el cuándo de la composición está dado por el cuándo de la vida.

No obstante, los criterios para definir cuándo algo ocurre y cuándo no ocurre pueden implicar vaguedad. Por ejemplo, Rea (1998) sugiere que los organismos son objetos compuestos en la medida que muestran algún tipo de funcionalidad, i.e., sus partes sirven para una función colectiva que las organiza en virtud de un todo unificado. Si la funcionalidad opera como un principio composicional, entonces una pregunta surge espontáneamente: “¿Hay algunos objetos que juntos muestren una organización funcional suficiente, tal que cuenten como partes de un todo, pero no partes de un organismo vivo?" (Rea, 1998, p. 354). Rea piensa

13 En una ontología cotidiana o de sentido común acerca de objetos físicos podemos decir, por ejemplo, que un montón de ladrillos claramente componen la Gran Muralla China, que el Obelisco de Buenos Aires con la Torre Eiffel en Paris claramente no componen un objeto juntos, y que no es claro del todo que muchas estrellas en el cielo compongan una constelación.

14 Esta tesis es también sostenida por Merricks (2001) pero por razones distintas, las cuales serán explicadas más adelante. Se puede revisar además Koslicki (2008, pp. 168-188) por otra teoría composicional restrictivista basada en la mereología aristotélica en la cual la composición ocurre cuando varios objetos componen un objeto de un cierto tipo (kind) de acuerdo con los componentes formales asociados a los objetos de ese tipo. 
que, aunque objetos organizados en forma de computador (computer-wise ${ }^{15}$ ) no componen cosas vivas, ellos sí satisfacen los criterios de funcionalidad y, por tanto, los de composicionalidad. Si esto es así, ¿puede haber entonces más objetos funcionalmente compuestos? Quizás las partes de un reloj están funcionalmente organizadas con una complejidad tal que, efectivamente, juntas componen un reloj. $\mathrm{O}$ bien, el planeta tierra puede ser considerado, en un sentido holista, como un objeto no vivo, pero cuya complejidad muestra una organización funcional de sus partes a partir de la actividad geológica, los ciclos naturales de las aguas, los periodos de glaciación y desglaciación, etc. El restrictivismo es, por lo tanto, una respuesta moderada a PSC en la que muchos objetos componen ocasionalmente un objeto, pero cualquier criterio para explicar el 'cuándo' de la composición puede ser poco preciso si encontramos casos límites de cuándo algunas cosas componen una. Por lo que, si existen muchos casos límite para nuestro criterio de composición, entonces hay buenas razones para considerarlo arbitrario. Sin embargo, este problema puede ser evitado si tomamos posiciones más radicales.

\section{Universalismo}

El 'nunca' y el 'siempre' son respuestas extremas y opuestas a PSC. Mientras el primero es defendido por el Nihilismo Composicional o Nihilismo Mereológico (o simplemente Nihilismo), el segundo es defendido por el Universalismo Composicional o Universalismo Mereológico (o simplemente Universalismo). El nihilismo, por un lado, es la tesis según la cual, para cualesquiera xs, nunca es el caso de que exista un objeto compuesto por los xs. El universalismo, por otro lado, es la tesis según la cual, para cualesquiera $x s$, donde los xs son objetos materiales, siempre es el caso que existe un objeto compuesto de los xs. Pese a que el nihilismo y el universalismo tienen diferentes compromisos ontológicos respecto a la composición material, ambos tienen una ventaja en común sobre el restrictivismo. Si la composición ocurre, ya sea nunca o ya sea siempre, entonces no hay cabida para la vaguedad de los casos límite. La vaguedad en la composición está vinculada con la idea de un límite metafísico que discrimine cuándo ocurre la composición y cuándo no ocurre (llamo a este límite el límite composicional). Si un límite composicional implica casos límites donde es indeterminado si algunos

15 Entiendo la expresión inglesa ‘wise' que se agrega a sustantivos que refieren objetos (como por ejemplo 'computer-wise') en el sentido de varios objetos dispuestos espacialmente de tal modo que sugieren la forma de $u n$ objeto determinado sin estar necesariamente comprometido con la existencia de este. 
objetos componen otros objetos, entonces el esfuerzo por fijar un límite preciso para restringir la composición, en un modo u otro, puede traer consigo distintos grados de vaguedad y arbitrariedad. No obstante, si la composición ocurre nunca o siempre, entonces no hay posibilidad ni de vaguedad ni de arbitrariedad. Consideremos primero el universalismo.

El universalismo parece ser una tesis muy extraña para ser aceptada por el sentido común. Si adoptas una ontología universalista, entonces existe un objeto compuesto por tu dedo pulgar izquierdo y el Cristo Redentor de Río de Janeiro (¡Literalmente!). Tal como Koons y Pickavance escriben: "Estas entidades que ni la ciencia ni el sentido común nunca han encontrado la necesidad de postular: el universalismo pareciera inflar innecesariamente nuestro inventario ontológico del mundo" (2015, p. 127). Si alguien está dispuesto a aceptar tal 'inflación innecesaria del inventario ontológico del mundo' debe ser por razones filosóficas bien justificadas. La más ampliamente aceptada es la necesidad de prevenir que la vaguedad y arbitrariedad se extiendan innecesariamente sobre el mundo. Como sabemos, el restrictivismo de sentido común es un principio composicional bastante impreciso y arbitrario. Por otro lado, un restrictivismo filosófico al estilo de van Inwagen puede traer consigo grados de vaguedad en la medida que el concepto de vida sea vago y existan casos límites en los cuales su uso no tenga una aplicación precisa. Por ejemplo, si algunas cosas no-vivas muestran una funcionalidad similar a la mostrada por cosas vivas, entonces nuestro principio de composicionalidad carece de un criterio claro. Así, la introducción de la vaguedad en el mundo parece ser inevitable. David Lewis tiene un pasaje famoso sobre esto:

\footnotetext{
El problema con una composición restringida es el siguiente: Es un asunto vago si una clase dada satisface o no nuestros desiderata intuitivos para la composición. [...] Pero si la composición obedece a una restricción vaga, entonces debe ser algunas veces una cuestión vaga si la composición tiene lugar o no. Y esto es imposible. La única tesis inteligible sobre la vaguedad la ubica en nuestro pensamiento y lenguaje. La razón de que sea vago dónde comienza el desierto no es que exista esta cosa ahí: el desierto, con bordes imprecisos; más bien, hay muchas cosas, con bordes diferentes, y nadie ha sido lo suficientemente tonto como para exigir que se tome una de ellas como el referente oficial de la palabra 'desierto'. La vaguedad es una indecisión semántica (1986, p. 212).
}

La fuente de la vaguedad está, de acuerdo con Lewis, en el lenguaje o el pensamiento, pero no en el mundo mismo. Si resulta ser vago si dos o más objetos componen algún objeto adicional no es debido a la indeterminación de un límite 
composicional, sino por los límites imprecisos de la extensión semántica de los conceptos que usamos o bien por la imprecisión de nuestras representaciones mentales. Una vez que estamos convencidos de la imposibilidad de límites composicionales ontológicamente vagos, la opción por el universalismo se presenta como inexorable. Los universalistas, de este modo, parecen estar de acuerdo con el siguiente argumento:

i. Si la composición está sujeta bajo algún tipo de restrictivismo, entonces podrían existir límites composicionales vagos (casos límites) en los cuales es indeterminado si la composición ocurre o no.

ii. No pueden existir límites composicionales vagos. De otro modo, el mundo mismo sería vago.

iii. Por lo tanto, si el mundo no puede ser vago, la composición debe ocurrir siempre.

Aunque el universalismo tiene muchas consecuencias filosóficamente problemáticas, ${ }^{16}$ este artículo no pretende una investigación de sus ventajas y desventajas. El objetivo principal es más bien destacar el rol que los límites pueden jugar en sus compromisos ontológicos. En este sentido, ya hemos notado que la motivación por adoptar el 'siempre' de la composición es, de hecho, un problema acerca límites. El universalismo está basado en la convicción filosófica de que no puede haber 'cortes' en la composición, puesto que cualquier corte repentino trae consigo vaguedad en el mundo respecto a los criterios que motivan el hacer un corte allí en vez de acá. Establecer cualquier 'corte' en la composición (i.e., un límite que separe cuándo la composición ocurre y cuándo no ocurre) involucra el trazado de un límite que acepta algunos casos en desmedro de otros. Sin embargo, en la medida que algunos casos límite (casos que no es claro de qué lado del límite trazado se ubican) surjan de ese corte composicional, podemos tener razones justificadas

16 Algunas de ellas son las siguientes. Por una parte, van Inwagen (1990) plantea que el universalismo sería similar a una teoría de conjuntos: para cualquier colección de objetos, existe un conjunto que siempre incluye esos objetos como sus miembros. El universalismo tendría, por tanto, compromisos con el esencialismo Mereológico (i.e., la tesis de que un todo está compuesto por sus partes necesariamente). Por otra parte, el universalismo es una tesis altamente contraintuitiva, ya que promueve la existencia de objetos demasiado extravagantes. Esto es lo que Markosian cataloga como la 'objeción fatal': "El universalismo implica que hay muchas más cosas compuestas de lo que las intuiciones de sentido común pueden permitir" (1998, p. 228). Podríamos tener buenas razones filosóficas para aceptar el universalismo, pero su promiscuidad ontológica, que va por lejos más allá del sentido común, hace difícil aceptar su tesis sobre la composición. 
para moverlo ligeramente más cerca o más lejos y, por ende, cambiar el corte o límite que trazamos en un comienzo. Podríamos entonces encontrar nuevas razones (también justificadas) para desplazar una vez más este nuevo límite y, así, la misma historia puede ser dicha acerca de cualquier límite o 'corte' en la composición. Si cada límite composicional trae consigo la existencia de casos límite, entonces la restricción de la composición implica siempre la existencia límites ontológicamente vagos. Hay tantos límites posibles (filosóficamente bien justificados) que tan solo escoger uno en lugar de otro sería una opción arbitraria. El universalismo rechaza la existencia de límites composicionales vagos y la arbitrariedad que conllevan, pero su solución es radical: no hay límites para determinar cuándo algunos objetos pueden componer un objeto adicional porque la composición sucede sin restricciones o es ilimitada —ocurre siempre.

La vaguedad aparece cada vez que pensamos en límites. Una vez que hemos eliminado los límites que restringen la composición, la vaguedad no es más un problema para estar ocupados. Si no hay tales límites, entonces no puede haber casos de vaguedad en nuestros criterios de por qué la composición ocurre en tal $y$ tal caso y no en tal y tal caso. Pensemos en las paradojas sorites. Un montón de arena puede sobrevivir la pérdida de algunos de sus granos si la porción apartada es lo suficientemente pequeña; el mismo montón no puede sobrevivir la pérdida de algunos granos si la porción apartada es lo suficientemente grande. Pero ¿cuándo 'suficientemente pequeño' y 'suficientemente grande' trazan un límite claro y preciso para determinar si el montón de arena ha sobrevivido o no la pérdida de alguno de sus granos? O bien, de igual manera, de los átomos que componen el Taj Mahal, ¿cuántos de ellos son necesarios para garantizar su existencia? ¿Cuál es el número de átomos para trazar el límite que indique cuándo sobrevive y cuándo es destruido dicho objeto? Hay tantos límites posibles para postular que cualquiera de ellos resultaría ser una preferencia completamente arbitraria. Así, hay diferentes grados de vaguedad en cualquier límite que pretenda restringir cuándo dos o más objetos componen un objeto o cuándo algo es capaz de resistir cambios mereológicos. Sin embargo, si el mundo mismo, de acuerdo con un universalista, no puede ser un lugar vago, es entonces preferible una ontología que descarte cualquier tipo de límite que discrimine casos donde la composición ocurra exitosamente y casos donde no. No hay límites metafísicos para la composición. Si hay límite alguno, este es trazado únicamente por el lenguaje y el pensamiento.

El 'siempre' del universalismo evita los problemas de vaguedad que trae consigo el 'ocasionalmente' del restrictivismo. Sin embargo, el universalismo es 
controversial puesto que no solo tolera átomos componiendo mesas o planetas, sino también objetos tales como aquel compuesto por la mesa donde almuerzas y Saturno (pues no hay límite alguno para la composición). Quizás, una respuesta mejor pero igual de radical a PSC puede ser el 'nunca' del nihilismo; la cual implicaría, no obstante, que no hay espacio en el mundo para la existencia de objetos ordinarios como mesas, planetas, o incluso tú mismo.

\section{Nihilismo: el problema de muchos (límites)}

El nihilismo ontológico (en el contexto de la tradición analítica de la metafísica) es la tesis de que no existe nada como tu casa, la cama donde duermes, los zapatos que vistes, y cualquiera de los objetos físicos ordinarios incluyendo tú mismo. ¿Cómo podemos darle sentido a tal postura radical ontológica llamada también eliminativismo ontológico? La estrategia parece paradójica: con el fin de rechazar la existencia del un gran número de cosas que el sentido común suele aceptar, un nihilista sostiene que existe un gran número de objetos también de una cierta clase. Los nihilistas no piensan que no haya nada en absoluto (lo cual sería absurdo). Ellos en cambio creen que no existe nada que esté compuesto de otras cosas. El mundo, así, consiste en un enorme número de simples o átomos mereológicos y nunca dos o más simples componen algo juntos — sin importar si ellos están lejos, cerca, muy cerca, o incluso cuando sus límites están en contacto.

¿Qué es lo que motiva a un nihilista a desechar las creencias del sentido común acerca del tipo de objetos que existen? A mi modo de ver, una motivación sigue lo que Peter Unger (1980) llama El Problema de Muchos (The Problem of Many), el cual básicamente proviene de la vaguedad encontrada en los límites de los objetos físicos ordinarios. Unger primero presentó este problema en dos trabajos diferentes en términos de 'sorites de descomposición': “There are no ordinary things" (1979) y más tarde en "I do not exist" (1997). El argumento es del siguiente modo:

i. Hay a lo menos una piedra.

ii. Para cualquier cosa que pueda existir, si es una piedra, esta consiste de muchos átomos, pero de un número finito.

iii. Para cualquier cosa que pueda existir, si es una piedra (consiste de muchos átomos, pero un número finito), entonces la eliminación neta de un átomo, 
o solo unos pocos, en un modo en el que sea lo más innocuo y favorable, no significará una diferencia en la medida que haya aún una piedra en la situación.

Estas tres premisas juntas son inconsistentes. Si hay una piedra, esta debe estar compuesta de un amplio número de átomos. Esto es, hay muchos, pero finitos átomos - digamos un billón - dentro de la región espacial $R$ ocupada por la piedra. La eliminación neta de un átomo de $R$ dejaría un billón menos un átomo en $R$ y aún la piedra ocupando $R$. Podemos extraer dos átomos de $R$ y sigue habiendo una piedra ocupando $R$, y así hasta extraer un billón de átomos de $R$. Lo absurdo es que no puede haber una piedra consistiendo de cero átomos. No puede haber una piedra ocupando $R$ donde ningún átomo es encontrado dentro de $R$. De acuerdo con Unger, la presunta existencia de la piedra en la premisa (i) nos ha llevado al absurdo en el que tenemos supuesta piedra que ocupa $R$ y que, paradójicamente, ningún átomo es encontrado en $R$. Si este argumento se aplica a piedras, mesas, planetas, y a cada objeto compuesto ordinario, entonces es mejor eliminar tales objetos de la lista de, parafraseando a Quine, lo que hay.

El problema de muchos está también basado en el sorites de composición, pero desde un enfoque distinto. El problema principalmente radica en la vaguedad de los límites de los objetos materiales ordinarios. La estrategia es pensar en la vaguedad que los conceptos sorites, tales como 'alto' o 'calvo', implican respecto a dónde un límite debe ser trazado. Ser alto o ser bajo tanto como ser calvo o ser no-calvo son características de las personas. ¿Cuál es sin embargo la altura exacta para determinar cuándo alguien es definitivamente alto en lugar de bajo o la cantidad exacta de cabello para determinar cuándo alguien es definitivamente calvo en lugar de no-calvo? ¿Hay límites exactos? Aparentemente, no hay sino límites difusos. Unger toma los límites de los objetos físicos ordinarios (superficies de las cosas) como instancias de vaguedad: "la idea principal es enfocarse en situaciones físico-espaciales donde ningún límite natural, lugar de parada natural, puede ser encontrado" (1980, p. 413). Tomando el ejemplo de una nube dado por Unger, detallaré a continuación el problema de los muchos.

Todo objeto físico ordinario es un objeto que tiene límites, i.e., la materia que lo compone alcanza un límite y, más allá de aquel, nada del objeto puede ser encontrado. Una nube es una instancia de objeto físico el cual debe tener un límite o, como Unger lo denomina, un lugar de parada (stopping place) donde "una vez que uno está más allá de tal lugar de parada, o grupo de lugares de parada, o, como 
lo llamo a menudo, de tal límite, uno está afuera de la nube, es decir, en un lugar donde la nube deja de ser" (1980, p. 419). Una nube, como cualquier objeto físico ordinario, tiene un límite como lugar de parada de la extensión de la porción de espacio físico ocupado por la materia que la compone.

Al ver a simple vista desde una larga distancia una nube blanca perfectamente formada aislada en un cielo azul, nuestro ojo pareciera reconocer en ella un límite claro que indica dónde la nube termina o se detiene (espacialmente) y dónde el cielo azul comienza (por ende, nada de la nube es encontrado). Si pudiésemos, sin embargo, mirarla muy de cerca (con algún aparato que nos permita observarla con más detalles), notaríamos que la esponjosa y homogénea materia que compone a la nube aparece, esta vez, como una gran colección de pequeñas gotas de aguas separadas unas de otras por extensas o menos extensas áreas de espacio vacío. En el límite o lugar de parada de la nube, la colección de gotas de agua y el cielo azul a su alrededor se mezclan y funden gradualmente: hay una transición progresiva de una mayor densidad a una menor densidad. Como Unger lo explica:

No existe un lugar absoluto donde repentinamente, o drásticamente, la 'densidad' se desvanece y la 'falta de densidad' primero comienza. [...] En esta realidad, la cual es, para todos los aspectos relevantes, la realidad efectiva, $[. .$.$] no hay un quiebre natural,$ o un límite, o un lugar de parada, para ser encontrado en cualquier nube (1980, p. 415).

La presunta nube ha sido hasta ahora considerada como un objeto único teniendo su propio límite. No obstante, el problema de muchos comienza justamente con la vaguedad involucrada al considerar objetos físicos ordinarios como entidades únicas teniendo un límite. Si continuamos con nuestra inspección detallada de la nube, notaremos que algunas gotas son definitivamente partes de esta (i.e., hay una certeza de que ellas pertenecen a la porción de espacio ocupada por la nube), mientras que para muchas otras gotas en los entornos de la nube es indeterminado si ellas son definitivamente partes del límite de la nube o definitivamente no lo son. Podemos, entonces, considerar como límite de la nube al tomar solo un grupo de estas gotas que están en esa zona indeterminada; pero si tomamos otro grupo de gotas de esa zona, el límite de la nube sería otro. Dado que existen muchos conjuntos de gotas que están en la línea fronteriza de la nube, hay también muchos límites posibles para la nube los cuales difieren sutilmente unos con otros. Por consiguiente, el presunto límite de la nube se revela ahora como un lugar de parada extremamente vago. El argumento de Unger puede ser simplemente parafraseado del siguiente modo: 
i. Si algo es una nube tipo, entonces hay algo que la delimita, i.e., algo que es el límite de la nube.

ii. En la vaguedad del lugar de parada de una nube existen millones de posibles candidatos para ser el límite de la nube, los cuales difieren sutilmente en el conjunto de gotas que los componen.

iii. Si hay millones de límites que son candidatos para ser el límite de la nube, entonces hay millones de nubes también (una para cada límite posible) donde solo una presunta nube existe.

¿Cuál es la nube original? ¿Cuál es el límite genuino entre todos los candidatos? Dada la sobrepoblación de límites, cualquier nube etiquetada como la 'original' sería una selección arbitraria. De modo tal que, si cada límite cuenta como un límite posible de la nube, entonces hay muchas colecciones de gotas que reclaman ser considerados como la presunta nube existente.

Ante esta situación paradójica, Unger ofrece una solución disyuntivista: o bien hay millones de límites posibles y, por tanto, candidatos genuinos para ser una nube, o bien no existe nube alguna. Dado que la primera opción lleva a un absurdo, la segunda es la más plausible. Si la existencia de los objetos físicos ordinarios y sus límites implica sobrepoblar el mundo con innumerables objetos innecesariamente, entonces es preferible desestimarlos de nuestra propuesta ontológica. Aunque el nihilismo y el universalismo son teorías filosóficas rivales, ambas están de acuerdo con un punto de vista: la composición no debería tener límites (o bien ocurre nunca o bien siempre, respectivamente) porque, de otra manera, el mundo sería un lugar absolutamente vago.

El problema de muchos es exclusivamente un problema de (muchos) límites. Una ontología de sentido común consiste de muchos objetos compuestos teniendo cada uno su límite o superficie. Sin embargo, la inclusión de tales límites en una ontología de objetos materiales introduce tal vaguedad en el mundo que incontables objetos comienzan a aparecer indiscriminadamente. Esto incita a un nihilista a adoptar una ontología radical en la cual la composición nunca acontece. Si no hay átomos que juntos componen una molécula de agua ni tampoco gotas de aguas que juntas componen una nube, entonces no hay nada que exista que sea una nube que posea algo así como un límite. El límite o lugar de parada de las cosas es donde todos los problemas ontológicos comienzan. 


\section{El argumento de la sobredeterminación}

Las únicas cosas que existen, sostendría un nihilista, son los simples, los que bajo ninguna circunstancia componen algo. Estos objetos son ontológicamente inocuos ya que no multiplican lo que hay innecesariamente. Este es el argumento que Theodore Sider llama 'Parsimonia Ideológica' el cual postula el siguiente principio epistémico: "Ideológicamente, las teorías más simples son más probables de ser verdaderas" (2013, p. 239). A diferencia del universalismo, el nihilismo es un ejemplo de parsimonia ontológica: el mundo consiste únicamente de objetos simples, esto es, no existen objetos que sean partes de otros objetos. En la medida que esta sea la propuesta ontológica correcta, entonces "el nihilismo nos permite eliminar 'parte' de la ideología de nuestras teorías fundamentales" (Sider, 2013, p. 240). Esta reducción de los componentes del mundo permite demostrar que paradojas como 'La Estatua y la Arcilla' o 'El Barco de Teseo' no son realmente un problema, puesto que no hay nada tal como estatuas, trozos de arcilla y barcos. Así, la ontología parsimoniosa del nihilismo es también defendida por Merricks (2001) en su argumento acerca de la sobredeterminación (overdetermination). Este consiste en las siguientes premisas:

i. Un objeto material ordinario $O$ es causalmente irrelevante si los $x S$ (los simples que componen $O$ ), actuando concertadamente, causan un evento físico $E$.

ii. $\quad E$ es causado por los $x s$.

iii. $E$ no está causalmente sobredeterminado.

iv. $\quad$ Si $O$ existe, no causa $E$.

v. Luego, si $\mathrm{O}$ existe, es causalmente inerte; carece de poderes causales.

Que un evento físico esté sobredeterminado implica la presencia de ciertas causas que no son requeridas para explicar cómo tal evento ocurre. Si un objeto o evento $x$ no es realmente requerido para la explicación causal de cualquier otro objeto o evento, entonces $x$ es innecesario: no existe. Por ejemplo, en el evento de una bola de béisbol que rompe una ventana, la bola no sería requerida para explicar el rompimiento del vidrio, ya que tal evento se encuentra ya explicado por átomos dispuestos en forma de bola quebrando el vidrio. La bola de béisbol (y cualquier 
objeto macro-físico que forman parte de una ontología de sentido común) sería simplemente un epifenómeno, pero nada físicamente relevante. Si la bola de béisbol no tiene poderes causales y, por ende, no hace contribución alguna para explicar el evento ocurrido, entonces no deberíamos aceptar su existencia, a menos que estemos dispuesto a aceptar que el rompimiento de la ventana está causalmente sobredeterminado. Debido a que no hay evento sobredeterminado, entonces la bola de béisbol no existe. De acuerdo con este argumento, nuestra ontología se debe mantener parsimoniosa con el fin de evitar una redundancia en la explicación causal de los eventos físicos. La opción nihilista al respecto es desestimar todo objeto hecho de partes, y así solo aceptar que los simples, actuando concertadamente sin componer nada, como expresa Merricks, son suficientes por sí solos para explicar los eventos físicos.

Respecto a los objetos físicos ordinarios, muchos de los fenómenos físicos que los afectan ocurren en sus límites o superficies. ${ }^{17} \mathrm{El}$ rompimiento de una ventana causado por una bola de béisbol ocurre cuando sus límites entran en contacto. Es más, el evento no es causado por todas las partes que componen la bola de béisbol ni todas las partes que componen la ventana, sino únicamente sus partes expuestas o superficies. Sin embargo, de acuerdo con el argumento de Merricks, si las causas de un evento físico pueden ser explicadas por átomos (dispuestos en formas de béisbol y ventana) que actúan concertadamente, entonces la existencia de objetos compuestos adicionales como bolas de béisbol y ventanas no es requerida. Si insistimos en que es justamente en las superficies de los objetos físicos ordinarios - sus límites - donde muchas de las interacciones físicas ocurren, entonces el mundo estaría innecesariamente saturado de objetos sobredeterminados causalmente.

Siguiendo la exposición del problema de los muchos de Unger, la aceptación de la existencia de un límite (de un objeto) — dada su vaguedad y borrosidadnos lleva a tener que aceptar la existencia de millones de límites (de millones de objetos). Así, el rompimiento de una ventana sería un evento sobredeterminado al estar causado por millones de posibles bolas de béisbol cada una teniendo su

17 Las superficies de las cosas son donde hacemos muchas operaciones físicas como pintar, rascar, rayar, pulir, raspar, lijar, etc.; o bien, ciertos eventos físicos como la reflexión de la luz o la corrosión de los metales también parecen ocurrir solo en las superficies. A su vez, muchas de las cualidades físicas que atribuimos a las cosas a través de nuestros sentidos acontecen sobre las superficies de estas: los colores de las cosas y sus texturas, por ejemplo, suelen ser percibidas en las superficies de las cosas. 
límite propio. Parece preferible, por lo tanto, eliminar los límites con el fin de evitar tales desastrosas consecuencias ontológicas. Los objetos físicos ordinarios y sus superficies son causalmente inertes; por ende, su existencia no es necesitada. La presunción de objetos compuestos y sus límites solo trae problemas ontológicos indeseables, los cuales, un nihilista sostendría, solo se solucionan si eliminamos tales entidades de nuestro inventario del mundo. Para esto es entonces necesario responder a PSC de modo radical: la composición nunca ocurre.

\section{Conclusión}

PSC es fundamentalmente una pregunta acerca de los límites y las consecuencias ontológicas de trazarlos. Hemos visto que tanto el universalismo como el nihilismo no aceptan ningún tipo de vaguedad ontológica en la composición material. La vaguedad es fundamentalmente una falta de claridad de por qué un límite debe estar aqui en lugar de allá: existen casos límite (borderline) donde es indeterminado si algo está definitivamente 'dentro' o definitivamente 'fuera' de lo que un límite separa. Tanto el universalismo como el nihilismo no aceptan que el mundo sea un lugar vago. La vaguedad de un límite solo puede ser el resultado de una indecisión semántica o una representación mental (o quizás la ignorancia de saber dónde se encuentra un límite claro). La respuesta restrictivista a PSC en la cual se establece un límite a la composición en el que ocasionalmente los objetos pueden componer otros objetos presenta el problema que trae consigo todo límite: ¿existe un lugar donde pueda un límite ser trazado objetivamente y sin arbitrariedad? Puesto que un límite parece siempre implicar diferentes grados de vaguedad, tanto el universalismo como el nihilismo descartan cualquier clase de límite o restricción para establecer cuándo la composición ocurre y cuándo no ocurre.

Tanto el universalismo como el nihilismo evitan la introducción de una vaguedad ontológica en la composición, pero difieren en la estrategia respecto a cómo trazar un límite que determine cuándo dos o más objetos componen un objeto adicional: mientras el universalismo sostiene que no existe tal límite (cualquier objeto compuesto está permitido), el nihilismo sostiene que existe tal límite (ningún objeto compuesto está permitido). Por un lado, el universalismo no está sujeto a la vaguedad composicional ya que no hay límites o restricciones de donde casos 'borderline' puedan surgir. Sin embargo, la desestimación de un límite para restringir cuando la composición ocurre nos lleva a la proliferación de límites de 
regiones espaciales ocupadas por una variedad de objetos compuestos que solo un universalista podría aceptar. Por otro lado, el nihilismo está comprometido con la tesis ontológica de un límite composicional tan rígido y bien definido que restringe cualquier clase de composición en el mundo: la composición nunca ocurre. Debido a este límite preciso e inamovible, los objetos físicos con los cuales tratamos en la cotidianidad y sus límites o superficies donde eventos físicos regularmente acontecen no son considerados componentes genuinos de la realidad. Así, dónde has trazado un límite para la composición determinará qué elementos aceptas como los componentes del mundo.

\section{Referencias}

Bolzano, B. (2014). Paradoxes of the Infinite. (D. A. Steele, Trad.). London: Routledge \& Kegan Paul.

Brentano, F. (1988). Philosophical Investigations on Space, Time and the Continuum. (B. Smith, Trad.). New York: Routledge.

Cartwright, R. (1975). Scattered Objects. En K. Lehrer(Ed.), Analysis and Metaphysics: Essays in Honor of R. M. Chisholm (pp. 153-171). Boston: D. Reidel Publishing Company.

Casati, R., \& Varzi, A. (1999). Parts and Places: The Structure of Spatial Representation. Cambridge MA: MIT Press.

Casati, R., \& Varzi, A. (2000). Topological Essentialism. Philosophical Studies: An International Journal of Philosophy in the Analytic Tradition, 100(3), 217-236.

Chisholm, R. (1976). Person and Object: A Metaphysical Study. Illinois: Open Court Publishing Company.

Gibbard, A. (1975). Contingent Identity. Journal of Philosophical Logic, 4(2), 187-221.

Hawley, K. (2014). Ontological Innocence. En A. J. Cotnoir, \& D. L. Baxter (Eds.), Composition as Identity (pp. 70-89). Oxford: Oxford University Press.

Johnston, M. (1992). Constitution is not Identity. Mind, 101, 89-105.

Koons, R., \& Pickavance, T. (2015). Metaphysics: The Fundamental. Oxford: Wiley Blackwell.

Koslicki. (2008). The Structure of Objects. Oxford: Oxford University Press.

Lewis, D. (1986). The Plurality of World. Oxford: Blackwell.

Lewis, D. (1991). Parts and Classes. Cambridge MA: Basil Blackwell.

Markosian, N. (1998). Brutal Composition. Philosophical Studies: An International Journal in the Analytic Tradition, 92(3), 211-249.

Merricks, T. (2001). Objects and Persons. Oxford: Oxford University Press.

Noonan, H. W. (1993). Constitution is Identity. Mind, 102(405) 133-145.

Rea, M. (1998). In Defense of Mereological Universalism. Philosophy and Phenomenological Research, 58(2), 347-360. 
Shoemaker, S. (1970). Wiggins on Identity. The Philosophical Review, 79(4), 529-544.

Sider, T. (2013). Against Parthood. En D. Zimmerman (Ed.), Contemporary Debates in Metaphysics (Vol. 8, pp. 237-293). Oxford: Blackwell.

Simons, P. (1987). Parts: A Study in Ontology. New York: Oxford University Press.

Unger, P. (1979). There are no Ordinary Things. Synthese, 41(2), 117-154.

Unger, P. (1980). The Problem of Many. Midwest Studies in Philosophy, 5, 411-467.

Unger, P. (1997). I do not Exist. En M. Rea (Ed.), Material Constitution: A Reader (pp. 175190). Oxford: Rowman \& Littlefield Publisher.

van Inwagen, P. (1990). Material Beings. London: Cornwell University Press.

Varzi, A. (1997). Boundaries, Continuity, and Contact. Noûs, 31(1), 26-58.

Varzi, A. (2016). Mereology. Stanford Encyclopedia of Philosophy, disponible en: https://plato. stanford.edu/entries/mereology/. Acceso el 5 April de 2018.

Wiggins, D. (1980). Sameness and Substance. Oxford: Basil Blackwell. 